\title{
Vehicle Target Recognition Algorithm based on Multidimensional Feature Fusion and Adaboost-SVM Strong Classifier
}

\author{
Shiqi WANG ${ }^{1}$ \\ ${ }^{1}$ Electrical \& Computer Engineering, University of Florida, USA
}

Keywords: Vehicle Target Recognition, Classifier, Image, Feature Fusion.

\begin{abstract}
Two image preprocessing methods have been introduced, comparative analysis has been made with combination of experiments; three traffic sign detecting methods have been summarized, including detecting algorithms based on color, shape and both; experimental comparative analysis has been made for the extraction methods for various traffic sign features, the experiment has proven that the probabilities of wrong identification for triangle and round marks are highest; after studying the features of existing traffic sign recognition method AdaBoost and SVM, One changing $\sigma$ AsaBoost technology, traffic sign detecting method combining color and shape, feature extraction method of sub-pattern combination has been adopted. On the basis of sub-model, it compares adjacent block, overlapped edge block and slide block method and distinguishes common traffic signs with the recognition method of support vector machines classifier combination based on radial basis function.
\end{abstract}

\section{Introduction}

The recognition of traffic signs is an important part of intelligent traffic signs. It involves sensor technology, information technology, automation technology, computer and many other technologies as well as how to distinguish roads, collisions, traffic signs and many other objects to be identified. Through years of researches made by domestic and foreign scholars, the recognition theory and technology system of traffic sign have attained breakthrough progress. Generally speaking, the image acquisition of traffic signs is the first step of realizing intelligent traffic. It is of great importance for the correctness and effectiveness of each operation in the following, but all the traffic signs are exposed in special outdoor environment. To make every driver see the traffic signs clearly, the traffic signs are usually set beside the road and at the turning. In these places, the traffic signs are easily affected by strong sunshine, dust and trees etc, so the clarity of image is very poor, and then it affects acquisition of camera for traffic signs. The image information received by built-in computer software and hardware system in the car is not clear. Because of this, people are devoting to studying how to improve the image recognition rate for traffic signs. This paper makes some related researches on how to improve the recognition rate of traffic signs. There is great distance between the research results and practical results, but the research process broadens horizons, increases knowledge and improves business level.

\section{Vehicle Target Recognition Algorithm}

Firstly, this paper introduces the experimental data of traffic mark recognition and understands the categories and features of traffic signs in the adopted data set. Secondly, it analyzes images in data set and adopts median filtering and Wiener filtering respectively to remove random noise and motion blur in image after comparing various results of image preprocessing algorithm, so that it can provide good image data for following traffic sign detecting and recognition.

The traffic sign image adopted in this paper is a game data set about computer intelligence advocated by IEEE world congress. It is collected and published by German Institute of Neural 
Computing. The purpose of the game is to develop intelligent recognition software that is suitable for Germany traffic sign detecting and recognition and provide service for safe traffic in Germany. This data set includes 43 categories of Germany traffic sign images with unified dimensions. The image data is collected under natural scene; it is affected by certain degree of sunlight and contains noise. This paper divides 43 categories of traffic signs into three categories based on the shapes of the signs. The first category is round shape (including 26 categories of traffic signs; at the same time, it classifies one eight sided shape signs into round shape), the second category is triangular shape (including 16 categories of traffic signs) and the third category is diamond shape (including only one type of traffic signs).

This data set has been published on the internet, in which the data is from a ten hours' video (it is collected from various roads in Germany during the day time in March 2010). The image acquisition equipment is a Prosilica GC $1380 \mathrm{CH}$ camera. The video frame rate is 25 frames/second; it is full-automatic exposure and the resolution of attained image is $1360 \mathrm{X} 1024$.

This data set includes a lot of track sequences. Each track sequence represents a traffic sign example. There are 30 traffic sign images in each track sequence and there are several to dozens of track sequences in each category of sample. Several track sequences combine a traffic sign sample, in which each traffic sign is unique and will not be repeated.

It is difficult to avoid various random noises as the traffic sign images are acquired in outdoor scene. In addition, during the acquisition, the camera on the vehicle keeps on moving, so certain motion blur will be produced to the image. We call the image polluted by the noise as degraded image. It can be expressed by formula as:

$$
g(x, y)=H[f(x, y)]+n(x, y)
$$

In which, $f(x, y)$ is original image, $n(x, y)$ is random noise, $H[]$ is degraded function, $g(x, y)$ is degraded image and the degraded process is as shown in the following:

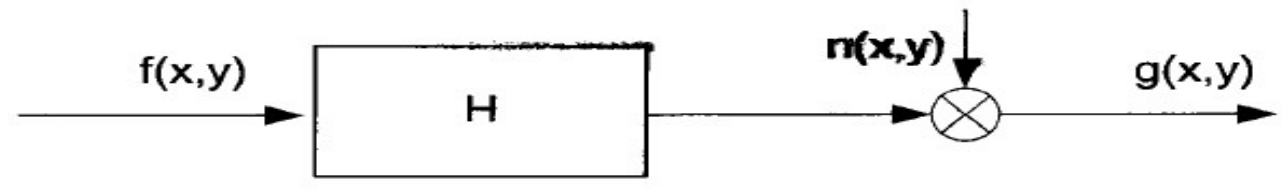

Fig.1. Image degraded process

In order not to affect image detecting and recognition, we need to preprocess it, including removing noise and motion blur. This chapter compares several image preprocessing algorithms. Finally, it confirms to adopt median filtering to remove random noise and adopt Wiener filtering to remove motion blur.

In the image preprocessing, the commonly used methods for removing random noise include median filtering, mean filtering and Gauss filtering etc.

1) Mean filtering

Mean filtering belongs to linear filtering, which is to set a window template for target pixel, and then remove all pixel gray values of target pixels within the window and replace gray value of original pixel with attained average value. Design reasonable window template, which is convolution kernel, it is the key of mea filtering. The common convolution kernel is as shown in formula (1):

$$
T^{3}=\frac{1}{9}\left[\begin{array}{lll}
1 & 1 & 1 \\
1 & 1 & 1 \\
1 & 1 & 1
\end{array}\right] \quad T^{3}=\frac{1}{5}\left[\begin{array}{ccc}
0 & 1 & 0 \\
1 & 1 & 1 \\
0 & 1 & 0
\end{array}\right] T^{3}=\frac{1}{25}\left[\begin{array}{lll}
1 & 1 & 1 \\
1 & 1 & 1 \\
1 & 1 & 1 \\
1 & 1 & 1 \\
1 & 1 & 1
\end{array}\right] T^{3}=\frac{1}{21}\left[\begin{array}{ccc}
0 & 1 & 0 \\
1 & 1 & 1 \\
1 & 1 & 1 \\
1 & 1 & 1 \\
0 & 1 & 0
\end{array}\right]
$$

2) Median filtering

In image processing, one common filtering method is median filtering. It is a non-linear sorting filter. Its principle is that sort all the pixels within $M \times N$ window based on gray values and select the median in the window after sorting to replace the gray value of original pixel. In this way, it gets 
closer to pixel of real value and then removes the noise.

Windows adopted by median filtering include square, cross and bar etc. For example, in one bar window, the gray values of each pixel are [3, 5, 201, 156, 6, 34, 67, 131, 167], and then the median of this window is 67 . The gray value of original pixel is replaced by 67 .

3) Gauss filtering

As a special linear mean filter, Gauss filtering is applied to the image de-noising process extensively and it can remove Gauss noise. Gauss filtering is to take Gauss function as weight function and weight the whole image to calculate mean value. The gray value of each pixel is the mean value calculated after weighting itself and gray value of pixels within the field. Common one-dimensional Gauss filtering function is:

$$
p(x)=\frac{1}{\sqrt{2 \pi \delta^{2}}} e^{\frac{-\left(x-x_{0}\right)^{2}}{2 \delta^{2}}}
$$

Core presentation layout of Gauss filtering is as shown in figure 2.

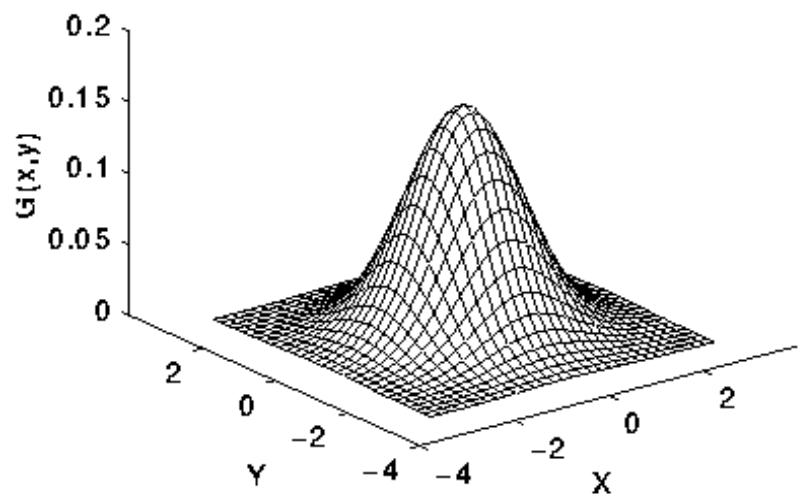

Fig.2. Core presentation layout of gauss filter

The basic principle of Gauss filter is to set a window template for target pixel, weight gray value of pixel within the template and calculate mean value, and then use this value to replace the gray value of original pixel.

\section{Experimental Results}

Figure 3 is the results of making mean filtering, median filtering and Gauss filtering for one traffic sign image respectively. It can be learnt through comparing the results of three filtering and principle of filtering algorithm that mean filtering is with fast execution speed, but it is easy to cause image blur. It does not remove random noise; instead, it spreads noise to other fields. Gauss filtering belongs to a special mean filtering; its filtering result is similar to the result of mean filtering; in addition, the bigger window template, the more blur image after filtering. Median filtering has one sorting process, so its calculation complexity is high, but it is not easy to cause image blur and the de-noising performance is very good. It can remove the random noise; at the same time, it will not make the image edge blur. To ensure the image processing after filtering as well as the accuracy of traffic sign recognition, we adopt median filtering to remove random noise. 

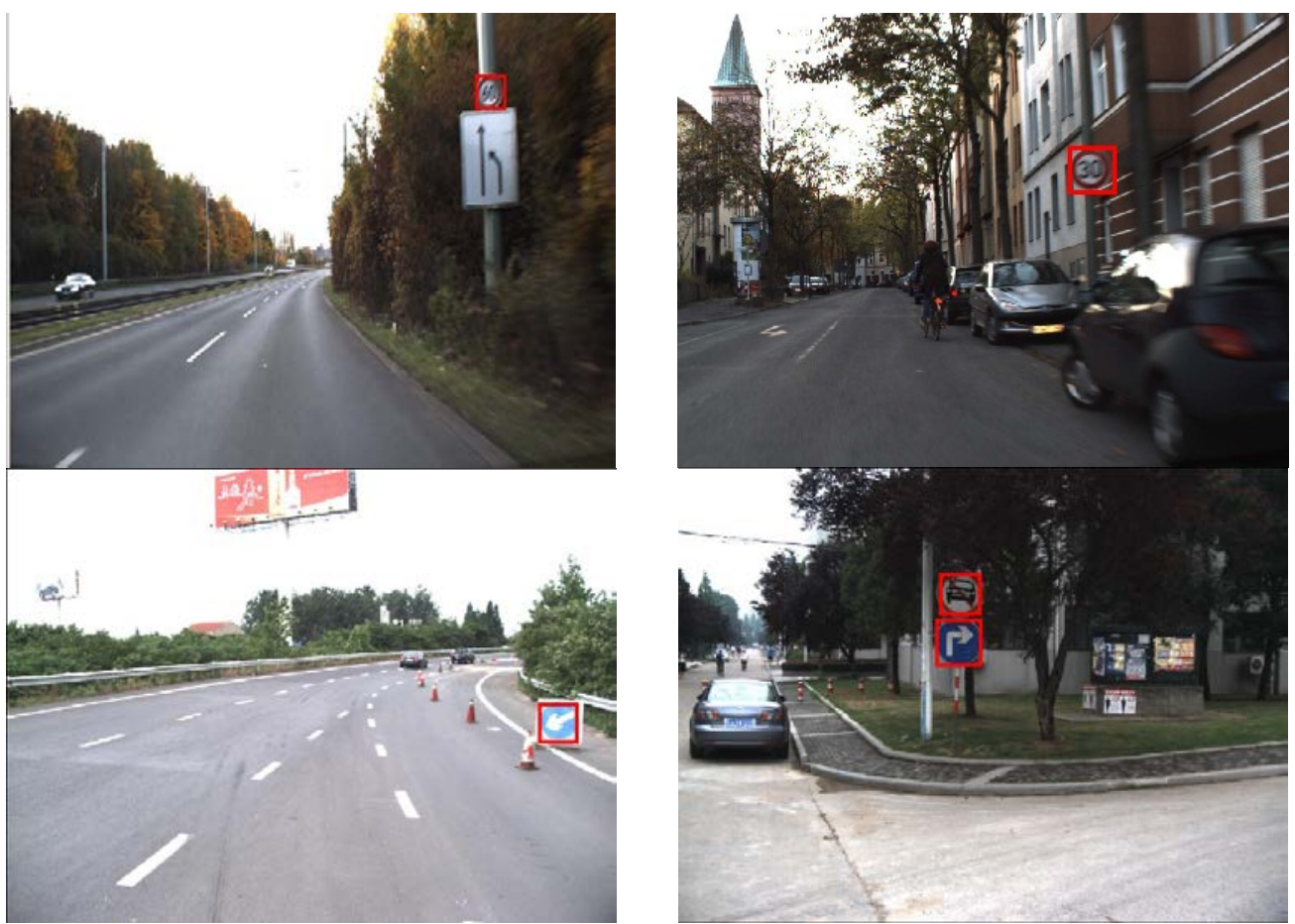

Fig.3. Traffic sign detecting results

\section{Conclusions}

This paper introduces several traffic sign detecting methods, including detection based on color space model, detection based on shape and detection based on color and shape. For the detection based on color space model, we introduce RBG color space model and HIS color space model. For RGB model, its application frequency in research is not high and the reason lies in its sensitiveness for sunlight. However, HIS color space model adopts non-linear conversion. Therefore, when the image is big, operation will bring great calculation burden to computer, which decreases traffic sign detection efficiency. For the detection method based on shape, the facts have proven that it is effective to detect traffic sign with shape. This method is with illumination robustness, but when the sign is deformed or covered, the success rate of detection is relatively low. In view of this, related experts and scholars have proposed a detection method combining color and shape together and made related experiments to verify the effectiveness of the method. This paper also adopts this method to detect traffic sign. Experiments have proven that this method is effective.

\section{References}

[1] Pahlavan K, Krishnamurthy P and Geng Y, Localization challenges for the emergence of the smart workd. IEEE Access, 2015, 3(1), pp. 3058-3067

[2] Lv, Z., Tek, A., Da Silva, F., Empereur-Mot, C., Chavent, M., \& Baaden, M. (2013). Game on, science-how video game technology may help biologists tackle visualization challenges. PloS one, 8(3), e57990.

[3] Lv, Z., Halawani, A., Feng, S., Li, H., \& Réhman, S. U. (2014). Multimodal hand and foot gesture interaction for handheld devices. ACM Transactions on Multimedia Computing, Communications, and Applications (TOMM), 11(1s), 10.

[4] Weisen Pan, Shizhan Chen, Zhiyong Feng. Automatic Clustering of Social Tag using Community Detection. Applied Mathematics \& Information Sciences, 2013, 7(2): 675-681. 